\title{
Regulation of Neutrophil Survival/Apoptosis by Mcl-1
}

\author{
Eric Milot ${ }^{1}$ and János G. Filep ${ }^{2}$ \\ ${ }^{1}$ Department of Medicine, Research Center Maisonneuve-Rosemont Hospital, \\ University of Montreal, 5415 Boulevard de l'Assomption, Montreal, QC, \\ Canada H1T 2M4 \\ ${ }^{2}$ Department of Pathology and Cell Biology, Research Center Maisonneuve-Rosemont \\ Hospital, University of Montreal, 5415 Boulevard de l'Assomption, Montreal, QC, \\ Canada H1T 2M4
}

Received 31 August 2011; Accepted 11 October 2011

Academic Editor: Marco Antonio Cassatella

Neutrophil granulocytes have the shortest lifespan among leukocytes in the circulation and die via apoptosis. At sites of infection or tissue injury, prolongation of neutrophil lifespan is critical for effective host defense. Apoptosis of inflammatory neutrophils and their clearance are critical control points for termination of the inflammatory response. Evasion of neutrophil apoptosis aggravates local injury and leads to persistent tissue damage. The short-lived prosurvival Bcl2 family protein, $\mathrm{Mcl}-1$ (myeloid cell leukemia-1), is instrumental in controlling apoptosis and consequently neutrophil lifespan in response to rapidly changing environmental cues during inflammation. This paper will focus on multiple levels of control of $\mathrm{Mcl}-1$ expression and function and will discuss targeting Mcl-1 as a potential therapeutic strategy to enhance the resolution of inflammation through accelerating neutrophil apoptosis.

KEYWORDS: Neutrophils, apoptosis, inflammation, resolution of inflammation, Mcl-1 (myeloid cell leukemia-1), gene transcription, proteasomal degradation, phosphorylation, caspase, MNDA (myeloid nuclear differentiation antigen), mitochondria, lipoxins, cyclin-dependent kinase inhibitors, $\beta 2$ integrin, formyl peptide receptors, sepsis 


\section{INTRODUCTION}

Neutrophils or polymorphonuclear leukocytes are the most abundant cells of the innate immune system and form the first line of defence against invading pathogens. Circulating neutrophils have a very short half-life because they constitutively undergo apoptosis and are functionally quiescent [1-3]. Neutrophil lifespan is dynamically influenced during the course of inflammatory response by multiple signals from the inflammatory microenvironment [3-5]. Recruitment of neutrophils into infected or injured tissues is associated with prolongation of their lifespan through delaying apoptosis, which allows performing their antimicrobicidal function effectively [6,7]. Shortened neutrophil survival enhances susceptibility to recurrent infections under some pathological conditions [8,9]. Elimination of the offending insult ideally prompts resolution of inflammation. Emigrated neutrophils undergo apoptosis and are removed by scavenger macrophages [10]. Excessive or dysregulated neutrophil responses together with inadequate repair contribute to persisting tissue damage that underlies many inflammatory diseases [2,11-13]. Efficient resolution of inflammation therefore relies on inhibition of neutrophil influx, recruitment of monocytes, rapid clearance of infiltrating neutrophils, and regeneration of damaged tissue structures $[14,15]$. Neutrophil survival/apoptosis emerged as one of the control points in resolving inflammation. Neutrophil apoptosis is tightly regulated by a complex network of signalling pathways that controls expression and degradation of key molecules, including Bcl-2 family proteins, activation of MAP kinases, NF- $\kappa \mathrm{B}$, and caspases $[1,3,4]$. One of the distinguishing features of neutrophil apoptosis is the preeminence of the Bcl-2 homolog Mcl-1 as a survival protein [16, 17]. In general, Mcl-1 expression inversely correlates with the degree of neutrophil apoptosis in both experimental models and clinical settings. Mcl-1 has some unusual properties compared with other Bcl-2 family members, including an extremely high turnover rate $[18,19]$, which is well suited for dynamic control of neutrophil survival. Understanding the molecular mechanisms that control Mcl-1 turnover and function may provide a rational basis for development of novel therapeutic approaches to modulate neutrophil apoptosis and consequently to facilitate resolution of neutrophil-mediated inflammatory diseases.

\section{NEUTROPHIL APOPTOSIS IN HUMAN DISEASE}

\subsection{The Fate of Neutrophils}

Under physiological conditions, circulating mature neutrophils are generally thought to have a lifespan $<1$ day in vivo $[20,21]$ though a recent study using in vivo labelling reported 10 times longer lifespan [22]. Circulating neutrophils are thought to undergo constitutive apoptosis and home to the spleen, liver, or back to the bone marrow to be destroyed by macrophages $[3,23]$. This mechanism is essential to keep the balance of cellular homeostasis [1]. Circulating neutrophils are rapidly recruited into infected or injured tissues. Outside-in signalling through the $\beta 2$ integrin Mac-1 during endothelial transmigration [24] or exposure of neutrophils to inflammatory mediators profoundly influences neutrophil survival and death $[1,3]$. Among the inflammatory mediators, granulocyte-macrophage colony-stimulating factor (GM-CSF) and IL-8, bacterial constituents, such as LPS and bacterial DNA containing unmethylated CpG motifs, or the acute-phase reactants $\mathrm{C}$-reactive protein and serum amyloid A can prolong the longevity of neutrophils, whereas proapoptotic stimuli, such as TNF- $\alpha$, TRAIL (TNF-related apoptosis-inducing ligand), or Fas ligand shorten their lifespan [25-29]. Phagocytosis of invading microorganisms triggers programmed cell death in neutrophils [30]. Apoptosis renders neutrophils unresponsive to extracellular stimuli, prevents release of toxic constituents, and facilitates their recognition and clearance by macrophages [10, 31]. Since, under most conditions, neutrophils will be exposed to multiple mediators, their fate would ultimately depend on the balance between prosurvival and proapoptotic cues (reviewed in [5]). Precise control of the neutrophil death program provides a balance between their defence functions and safe clearance, whereas impaired regulation of neutrophil death is thought to contribute to a wide range of inflammatory pathologies $[2,11,12]$. 


\subsection{Altered Neutrophil Survival/Apoptosis in Human Disease}

Neutrophil death is tightly regulated on a constitutive basis, and the complexity increases under pathological conditions, so that it is often difficult to decide whether survival or apoptosis is most favourable from the host's perspective. Indeed, both accelerated and delayed neutrophil apoptosis could have severe pathological consequences. For instance, pyocyanin produced by the opportunistic pathogen Pseudomonas aeruginosa [32], influenza virus A [33], and HIV [8] shortens neutrophil longevity by accelerating apoptosis, leading to impaired antimicrobial defences and increased susceptibility to recurrent infections. Upregulation of Fas is thought to contribute to accelerated neutrophil apoptosis in patients with systemic lupus erythematosus [34].

A wide variety of inflammatory diseases, including acute respiratory distress syndrome (ARDS), pneumonia, sepsis, acute coronary artery disease, rheumatoid arthritis, and cystic fibrosis, is associated with delayed neutrophil apoptosis in the blood, sputum, or synovial fluid [35-40]. A frequent finding in these studies is the correlation of neutrophil apoptosis with the severity and/or outcome of the disease. Various mechanisms have been implicated in suppression of neutrophil apoptosis. For example, enhanced GM-CSF production is thought to prolong neutrophil survival in sepsis [35], severe burns [36], ARDS [37], or acute coronary artery disease [38], whereas NF- $\kappa$ B-mediated survival cues have been implicated in chronic obstructive pulmonary disease and respiratory syncytial virus infection-associated delay of neutrophil apoptosis [39, 40]. Lactoferrin released from activated neutrophils [41] and serum amyloid A [42] have been implicated in increased neutrophil longevity in rheumatoid arthritis. Resistance to Fas ligand-triggered apoptosis and direct inhibition of caspase-3 by acrolein, a toxic unsaturated aldehyde found in cigarette smoke, has also been implicated in suppression of neutrophil apoptosis [43, 44]. Intriguingly, intracellular pathogens may use apoptotic neutrophils as a Trojan horse to promote infection [45, 46].

\subsection{Bcl-2 Family Proteins in Human Neutrophils}

Neutrophils constitutively express the proapoptotic members of the Bcl-2 family, including Bax, Bad, Bak, Bid, and Bik [47]. These proteins have relatively long half-lives, and their cellular levels change very little during exposure of neutrophils to agents that either accelerate or delay apoptosis. Human neutrophils also express the antiapoptotic proteins $\mathrm{Mcl}-1, \mathrm{~A} 1$, and to a much lesser extent $\mathrm{Bcl}-\mathrm{X}_{\mathrm{L}}$, but not Bcl-2 [48]. Mcl-1 and to a lesser extent A1 appear to be important for maintaining cytokine-regulated survival $[17,49]$ and have been implicated in signalling extended neutrophil lifespan in response to a variety of proinflammatory stimuli [50-52]. Survival of myeloid cells markedly decreases following treatment with antisense oligonucleotides against Mcl-1 [53]. Increased Mcl-1 expression has been detected in neutrophils isolated from patients with Crohn's disease [54] and severe sepsis [55].

\section{REGULATION OF NEUTROPHIL APOPTOSIS BY Mcl-1}

The mechanisms that govern neutrophil death have been extensively reviewed $[3-5,56]$. Apoptosis may result from activation of the extrinsic, intrinsic, or endoplasmic reticulum stress pathways [3,57]. A complex network of intracellular death/survival signaling pathways regulates neutrophil apoptosis, and the balance of these circuits would ultimately determine the fate of neutrophils. There is now evidence that suggests existence of hierarchy among these signals [5, 57]. For instance, ligation of Mac-1 integrates life and death decisions in a ligand-specific manner [11,30]. Mac-1-mediated phagocytosis of opsonized bacteria triggers apoptosis [58, 59], whereas the binding of Mac-1 to ICAM-1, fibrinogen [30], or myeloperoxidase [11] prolongs the lifespan of neutrophils by suppressing apoptosis. Likewise, the pleiotropic receptor formyl peptide receptor 2 (formyl-peptide-like-1 receptor/lipoxin receptor or FPR2/ALX) [60] generates opposing signals to suppress or activate the apoptotic machinery following binding of one of its many ligands, including serum amyloid A, the anti-inflammatory lipids lipoxin $\mathrm{A}_{4}$ and aspirin-triggered 15-epi-LXA , annexin A1, and the antimicrobial cathelicidin peptide human CAP18/LL37 [27, 60-63]. Many survival and proapoptotic signalling pathways converge to influence expression of Mcl-1, which is central for 
maintaining neutrophil survival. Loss of Mcl-1 together with direct activation of the proapoptotic protein Bax by calpain-1 [64] is likely responsible for rapid apoptosis. Indeed, neutrophil aging is associated with decreased expression of calpastatin, a specific inhibitor of calpain-1, resulting in increased calpain1 activity.

\subsection{Properties of $\mathrm{Mcl}-1$}

The human Mcl-1 gene is located on chromosome 1q21 and comprises three exons [65, 66]. The prototypical Mcl-1 protein (also referred as $\mathrm{Mcl}-1_{\mathrm{L}}$ ) comprises 350 amino-acid residues and contains three BH (Bcl-2 homology) domains (BH1-3), which confer the ability to heterodimerize with other family members [67]. Unlike Bcl-2 and Bcl- $\mathrm{X}_{\mathrm{L}}, \mathrm{Mcl}-1$ and $\mathrm{A} 1$ lack the $\mathrm{N}$-terminal BH4 domain. Mcl-1 contains a C-terminal transmembrane domain that serves to localise Mcl-1 to the mitochondrial outer membrane and other intracellular membranes [68]. The N-terminal region contains PEST domains, rich in proline $(\mathrm{P})$, glutamic acid $(\mathrm{E})$, serine $(\mathrm{S})$, and threonine $(\mathrm{T})$, and four Arg:Arg motifs that target Mcl-1 for rapid turnover by the proteasome. Indeed, the half-life of the mature protein is estimated to be between 1 and 5 hours $[69,70]$.

Mcl-1 promotion of neutrophil survival is thought to involve heterodimerisation with and neutralisation of proapoptotic Bcl-2 family proteins Bim or Bak in the mitochondrial outer membrane $[71,72]$. This leads to suppression of cytokine $\mathrm{c}$ release from mitochondria and Apaf-1-dependent activation of caspase-3. Loss of mitochondrial transmembrane potential $\left(\Delta \Psi_{m}\right)$ precedes development of apoptotic morphology in neutrophils undergoing constitutive [73] or induced apoptosis.

\subsection{Transcriptional Control of Mcl-1 Expression}

The promoter region of Mcl-1 contains an array of putative and confirmed transcription binding sites, including consensus STAT response elements, cAMP response elements (CRE), and NF- $\kappa$ B binding sites [74]. In a variety of cancer cells, transcription of Mcl-1 has been reported to be induced by cytokines, such as IL-3, IL-5, IL-6, and granulocyte-macrophage colony-stimulating factor (GM-CSF), growth factors, and hypoxia-inducible factor $1 \alpha$ under hypoxic conditions (reviewed in [66]). GM-CSF also stimulates Mcl-1 transcription in human neutrophils, but these changes were disproportionately small compared with changes at the protein level $[47,74,75]$, suggesting a limited role for transcriptional regulation of Mcl-1 in cytokinemediated neutrophil survival. GM-CSF does not appear to signal through NF- $\kappa$ B [76]. G-CSF continues to affect longevity of mature neutrophils at transcriptional level during in vivo mobilization of granulocytes for transfusion purposes [77]. Hypoxia has been reported to delay neutrophil apoptosis $[52,78,79]$ though the role for HIF- $1 \alpha$ modulation of Mcl-1 transcription remains to be investigated. NF- $\kappa$ B has been proposed to play an important role in neutrophil survival [80], but curiously, there is no evidence for NF- $\kappa \mathrm{B}$ regulation of Mcl-1 transcription in these cells.

Downregulation of Mcl-1 mRNA is largely due to inactivation of transcription factors that stimulate Mcl-1 transcription [66]. Another mechanism is direct repression of the Mcl-1 promoter by the transcription factor E2F1, a key cell cycle regulator [81]. However, the function and activation of E2F1 in neutrophils are largely unknown.

\subsection{Posttranscriptional, Translational, and Posttranslational Control}

In addition to transcriptional regulation, Mcl-1 is also subject to posttranscriptional, translational, and posttranslational control. By removing exon 2, alternative splicing of Mcl-1 mRNA produces a shortened form of Mcl-1, Mcl-1s, which lacks BH1, BH2, and the transmembrane domain [66]. Another splice variant, Mcl-1 $1_{\mathrm{ES}}$, lacks a portion of exon 1 but retains all three $\mathrm{BH}$ domains and the $\mathrm{C}$-terminal transmembrane domain [82]. These splice variants are unable to bind and sequester proapoptotic Bcl-2 family members; indeed, they induce apoptosis by inhibiting full-length Mcl-1 [83]. Accumulation of $\mathrm{Mcl}-1_{\mathrm{S}}$ has been 
detected in macrophages during bacterial clearance, and this appears to regulate macrophage commitment to apoptosis, likely facilitating the resolution of inflammation [84]. At the present time, no information is available whether $\mathrm{Mcl}-1_{\mathrm{S}}$ is also generated in aging neutrophils.

The rate of Mcl-1 mRNA transcription is tightly regulated, and Mcl-1 mRNA, like the protein, has a very short half-life [66]. Enforced expression of the microinhibiting RNA 29b (Mir-29b) was found to reduce Mcl-1 translation and to induce apoptosis in malignant cholangiocarcinoma cells [85].

Posttranslational modifications can also modify the stability and function of Mcl-1. Sequence analysis and mutational analysis of Mcl-1 revealed several potential phosphorylation sites in the PEST domains, which may mediate complimentary or opposing processes (reviewed in [66]). For instance, cyclindependent kinase 1 (CDK1), CDK2, or JNK-mediated phosphorylation of Ser ${ }^{64}$ appears to be required for dimerisation with other Bcl-2 homologues and antiapoptotic function without affecting stability [86]. Phosphorylation of $\mathrm{Thr}^{92}$ (in conjunction with $\mathrm{Thr}^{163}$ ) by ERK-1 stabilizes Mcl-1 and is likely required for antiapoptotic function [87]. Glycogen synthase kinase-3 (GSK3) phosphorylates Ser ${ }^{155}$, in conjunction with $\mathrm{Ser}^{159}$ and $\mathrm{Thr}^{163}$, resulting in destabilization and impaired antiapoptotic function of Mcl-1 [88-90]. Ser $^{121}$ is a target for JNK and p38 MAPK, and phosphorylation of this residue has been reported to either inactivate or stabilize Mcl-1, most likely representing differences in the cell lines studied. One should recall that since most of these studies were performed in tumor cells, it is uncertain whether these mechanisms are also operational in neutrophils. Contradictory results have been reported for p38 MAPK in human neutrophils; its action on neutrophil survival may be stimulus and/or context specific (reviewed in [48]). Prosurvival function of p38 MAPK may include phosphorylation and inactivation of caspase- 3 and caspase8 [91], whereas in other studies, constitutive neutrophil apoptosis was found to be associated with prolonged phosphorylation of p38 MAPK [27, 28, 92].

\subsection{Intracellular Localisation}

Under resting conditions Mcl-1, is localized to the cytoplasm, presumably as a heterodimer with Bax and/or Bad, the outer mitochondrial membrane [68], the nuclear envelop [52], and the nucleus [93, 94]. Concomitant activation of the MEK/ERK 1/2 and phosphoinositide-3-kinase (PI3 K)/Akt pathways by proinflammatory mediators or ligation of Mac-1 [11, 27, 48, 92] results in phosphorylation of Bax and $\mathrm{Bad}$, leading to dissociation of Mcl-1 from phosphorylated Bad or Bax in the cytoplasm. This permits targeting of Mcl-1 to the mitochondria, where it counters the activity of Bak and Bim [95]. Akt-mediated phosphorylation of Bax at $\mathrm{Ser}^{184}$ promotes heterodimerization with Mcl-1 or A1 [96]. Interaction of Mcl1 with CDK1 and proliferating cell nuclear antigen (PCNA) is thought to be responsible for its nuclear localisation in proliferating cells $[93,94]$.

\subsection{Signals from the Nucleus}

Nuclear factors could modulate execution of death program by different ways. While some nuclear factors such as E2F1, STAT3, STAT5, HIF- $1 \alpha$, and NF- $\kappa$ B control expression of genes encoding pro- or antiapoptotic factors, other nuclear proteins could act as nuclear transducers and influence the extrinsic or intrinsic apoptotic signaling pathways following their relocalisation to the cytoplasm. While p73 was suggested to exert such activity [97], the cytoplasmic accumulation of the nuclear proteins p53, p21/WAF1, nur77, and SHP has been shown to directly affect pro- or antiapoptotic factors, and hence, apoptosis in cancer cells [98-102]. Recent studies have identified important roles for the nuclear proteins PCNA (proliferating cell nuclear antigen) and MNDA (myeloid nuclear differentiation antigen) in the regulation of constitutive apoptosis in human neutrophils [55, 103].

In proliferating cells, PCNA is predominantly located in the nucleus where it influences DNA replication, transcription, cell cycle, chromosomal segregation, and DNA repair processes [104]. In neutrophils, PCNA accumulates in cytoplasm where it physically interacts with and sequesters procaspase- $3,-8,-9$, and -10 [103]. Cytoplasmic accumulation of PCNA decreases rapidly in aging neutrophils, and interestingly, it increases in neutrophils exposed to the survival signal G-CSF [103]. 
Cytoplasmic accumulation of MNDA has been reported to influence the fate of Mcl-1 both in human neutrophils and in HL-60 cells [55]. MNDA, a member of the human HIN-200 family, exhibits characteristic nucleolar localization and is thought to be a myeloid-specific factor possibly involved in myeloid cell differentiation [105]. MNDA relocates to the cytoplasm, and it is cleaved by caspases, presumably caspase3 in neutrophils undergoing constitutive apoptosis and HL-60 cells exposed to genotoxic stress [55]. MNDA directly interacts with Mcl-1, promotes its proteasomal degradation, and consequently leads to collapse of mitochondrial transmembrane potential [55]. Furthermore, in neutrophils from patients with severe sepsis, cytoplasmic relocalization of MNDA is impaired which correlates with delayed apoptosis and appears to portend a poor prognosis for septic patients. LPS, bacterial DNA, and platelet-activating factor, which activate survival signaling circuits in neutrophils during severe sepsis, prevent cytoplasmic accumulation of MNDA parallel with suppression of apoptosis [55]. While hindrance of mitochondrial dysfunction and consequently attenuation of caspase- 3 activation likely contribute to impaired MNDA translocation, additional work is needed to explore the underlying molecular links. Knockdown of MNDA in HL-60 cells rendered HL-60 cells resistant to genotoxic stress-induced apoptosis [55].

Taken together, these studies suggest a complex role for nuclear proteins in the regulation of constitutive neutrophil apoptosis. Indeed, while MNDA relocalization to the cytoplasm facilitates apoptosis, the cytoplasmic accumulation of PCNA is associated with delayed neutrophils apoptosis. Noticeably, the downstream "apoptosis-controlling" proteins targeted by PCNA and MNDA are also different.

\subsection{Mcl-1 Degradation}

Beside specific kinases implicated in the phosphorylation of Mcl-1, the E3 ubiquitin ligase MULE (Mcl1 ubiquitin ligase E3) is responsible for the constitutive polyubiquitination of $\mathrm{Mcl}-1$ and subsequent proteasomal degradation [106]. GM-CSF promotion of neutrophil survival is predominantly mediated through blocking this process [75]. Conversely, the deubiquitinase USP9X reverses polyubiquitination of Mcl-1 and promotes its stability [107]. Since MNDA does not appear to interact with any of these factors (unpublished observations), it is not clear how and when, during the multiple steps leading to Mcl-1 proteasomal degradation, MNDA interferes with this event. It remains to be defined why a cell-specific protein, like MNDA, exerts such a critical function on Mcl-1 turnover, while Mcl-1 is critical for survival of many other cell types that do not express MNDA.

Mcl-1 degradation during apoptosis may be further accelerated by activation of other E3 ubiquitin ligases and/or cleavage by other proteases [66]. For instance, caspase-3-mediated cleavage of Mcl-1 within the N-terminus has been reported [108, 109], which may convert Mcl-1 to a proapoptotic protein [108]. Intriguingly, in high-purity human neutrophils, initial falls in Mcl-1 level precede caspase-3 activation and apoptosis, whereas at later time points, drops in Mcl-1 can be reversed with caspase inhibition [110]. These observations suggest that Mcl-1 may also function as an upstream regulator of caspase activation in addition to being a target for degradation by caspases.

\section{Mcl-1 AS A THERAPEUTIC TARGET}

Targeting the apoptotic machinery in neutrophils has emerged as a potential approach to promote resolution of inflammation. Neutrophil apoptosis is essential for their clearance from inflamed tissues. Apoptotic neutrophils stop releasing proinflammatory mediators, secrete alpha-defensins [111], and sequester cytokines $[112,113]$. Phagocytosis of apoptotic neutrophils and other cells induces macrophages to switch from a proinflammatory to a proresolution phenotype [114], thereby activating proresolution circuits [15]. The importance of Mcl-1 as a key regulator of neutrophil survival renders this protein a promising target for therapeutic induction of apoptosis in inflammatory neutrophils. Indeed, therapeutic strategies to redirect neutrophils to apoptosis appear to be mediated, at least in part, through modulation of Mcl-1.

Pharmacological inhibition of ERK1/2 and PI3K signalling has emerged as a potential resolution therapy. PI3K has been implicated in persistence of neutrophil-mediated inflammation [115]. Selective 
ERK1/2 blockade was reported to enhance resolution of inflammation and was linked indirectly to an increase in neutrophil apoptosis in a mouse pleurisy model [116]. Interestingly, treatment of neutrophils with sodium salicylate results in p38 MAPK-mediated reduction of Mcl-1 expression and acceleration of apoptotic cell death [117]. The phosphodiesterase 4 inhibitor, rolipram, reduces neutrophil accumulation and induces neutrophil apoptosis in LPS-induced pleurisy in mice [118]. Resolution of pleural inflammation is associated with inhibition of the PI3K/Akt pathway, coinciding with decrease in Mcl-1 protein levels in neutrophils, and can be blocked by the pan-caspase inhibitor zVAD-fmk. Although rolipram also inhibited $\mathrm{NF}-\kappa \mathrm{B}$ activation in infiltrating leukocytes, this pathway does not appear to be relevant to resolution, for other NF- $\kappa$ B inhibitors, pyrrolidine dithiocarbamate (PDTC), and SN50 failed to reduce neutrophil accumulation in the pleural cavity [118].

Inhibition of survival signals generated by outside-in signalling through Mac-1 has also been implicated in mediating the anti-inflammatory and proresolution actions of lipoxin $\mathrm{A}_{4}$ and aspirin-triggered 15epi-LXA 4 . Thus, 15-epi-LXA 4 administered intravenously as a bolus injection at the peak of inflammation enhances resolution of exogenous and endogenous MPO-mediated pulmonary neutrophil accumulation and acute lung injury and improves the survival rate of mice [119]. 15-epi-LXA 4 attenuates MPO activation of ERK1/2 and PI3K and redirects neutrophils to apoptosis by decreasing Mcl-1 expression [119]. Conversely, MPO-deficient mice exhibit reduced tissue neutrophil accumulation and injury following injection of live E. coli [120] and during ischemia/reperfusion [121]. It is not known whether MPO deficiency could affect Mcl-1 expression and the longevity of neutrophils. Aspirin or lovastatin reduction of acid aspiration-induced lung inflammation is, in part, mediated through stimulation of 15 -epi-LXA $4[122,123]$ though the effect of lovastatin on neutrophil apoptosis in this model has not been investigated. In vitro, aspirin or sodium salicylate counteracts LPS-, but not GM-CSF-mediated neutrophil survival [76] through inhibition of NF$\kappa \mathrm{B}$ activation [124] and acceleration of Mcl-1 degradation [117, 125]. Consistently, both aspirin and sodium salicylate increase the percentage of apoptotic neutrophils in thioglycollate-induced peritonitis [76].

Similar to lipoxins, resolvin E1 and D2, derived from the enzymatic modification of $\omega-3$ polyunsaturated fatty acids $[15,126]$, were reported to effectively attenuate excessive neutrophil trafficking in acid aspiration or E. coli-evoked pneumonia [127] and cecal ligation and puncture-induced peritonitis [128]. The impact of resolvins on neutrophil apoptosis remains to be investigated.

Another possibility to induce neutrophil apoptosis is the use of CDK inhibitors, in particular Rroscovitine, to accelerate Mcl-1 degradation [129]. Human neutrophils express CDK1, CDK2, and CDK5 $[129,130]$ though their function in terminally differentiated neutrophils is largely unknown. CDK1 phosphorylation of $\mathrm{Ser}^{64}$ in Mcl-1 enhances its antiapoptotic action [86] but does not appear to affect the stability of Mcl-1 [66]. Regardless of the precise molecular mechanism of action, R-roscovitine was reported to enhance resolution of pleural inflammation [129], bleomycin-induced lung injury [129], arthritis [129], and hemorrhagic brain damage in mice [131] presumably through accelerating caspase-dependent neutrophil apoptosis. In vitro culture of neutrophils form patients with cystic fibrosis with R-roscovitine restores impaired apoptosis to normal level [132] though the underlying mechanisms remain to be investigated.

Neutrophils may remain sensitive towards Fas ligand- or TNF-triggered apoptosis despite of suppressed intrinsic pathway. Thus, ex vivo cross-linking Fas with agonistic anti-Fas IgM on neutrophils from severely injured patients, which express high levels of Mcl-1, was found to evoke caspase-mediated Mcl-1 cleavage and mitochondrial dysfunction [133].

While these observations clearly indicate that ablating Mcl-1 expression and/or function is sufficient to promote apoptosis in neutrophils, nonselective targeting of Mcl-1 may have potential toxicity in lymphoid tissues, as suggested by Mcl-1 knock-out experiments [134, 135]. Selective Mcl-1 antagonism in inflammatory neutrophils would likely overcome such limitations.

\section{CONCLUSION}

Neutrophil apoptosis has emerged as an important control point in determining the outcome of the inflammatory response. Thus, suppressed neutrophil apoptosis contributes to persisting inflammation, 


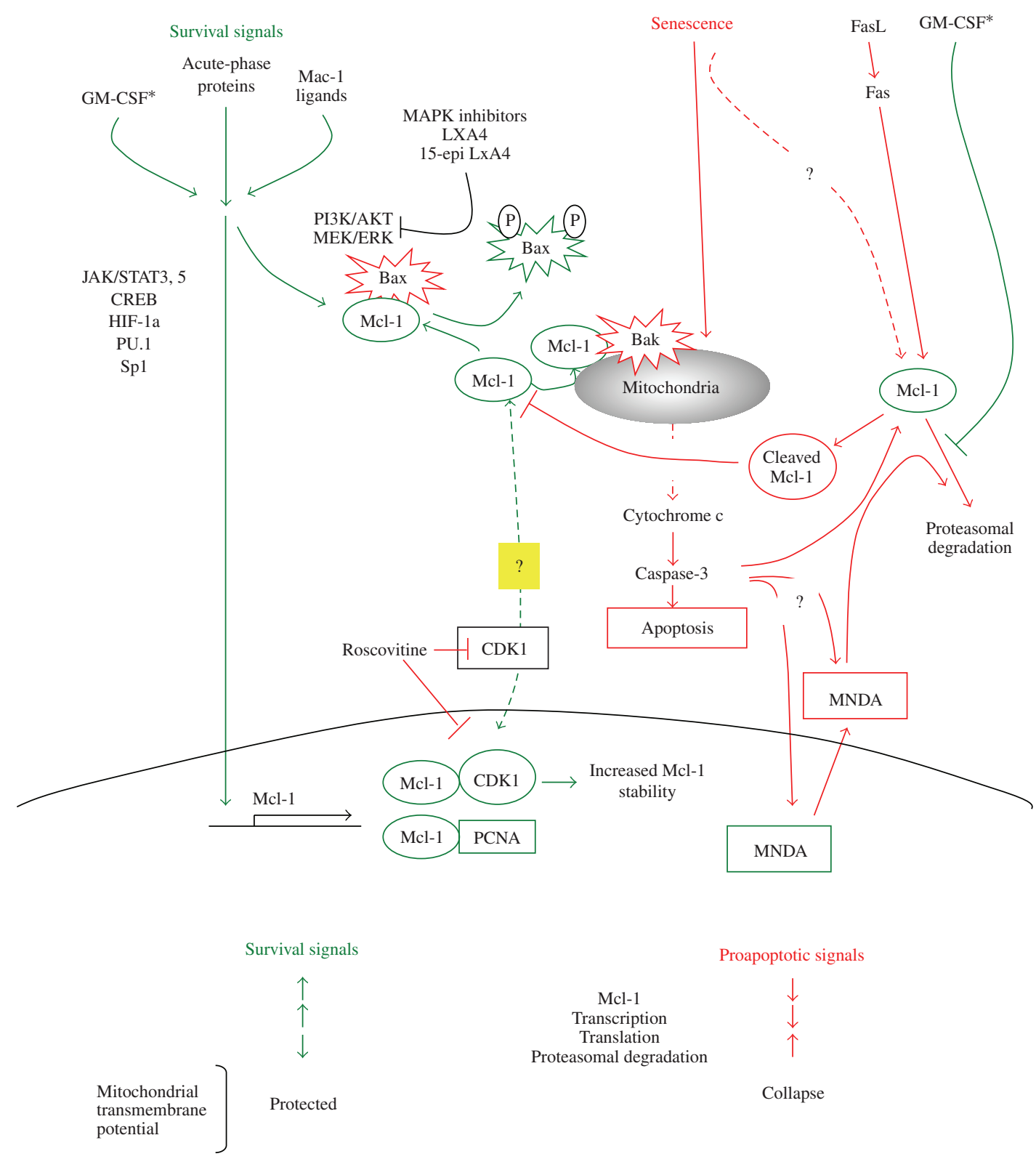

FIGURE 1: Mcl-1 integrates survival and proapoptosis cues in human neutrophils. Mcl-1 functions as a survival signal for human neutrophils by blocking the proapoptotic action of Bak (and/or Bim) at the outer mitochondrial membrane. In aging neutrophils, decreases in $\mathrm{Mcl}-1$ level may precede initiation of the cell death program. At later stages, $\mathrm{Mcl}-1$ is rapidly degraded by the proteasome and/or cleaved by caspase-3. Ligation of Fas accelerates Mcl-1 proteasomal degradation. Falls in $\mathrm{Mcl}-1$ lead to collapse of mitochondrial function, and apoptosis. Cytoplasmic accumulation of the nuclear protein MNDA further accelerates Mcl-1 degradation. Survival cues from GM-CSF, acute-phase proteins, and ligation of Mac1 have been reported to enhance transcription of $\mathrm{Mcl}-1$, promote dissociation of $\mathrm{Mcl}-1$ from Bax (and/or $\mathrm{Bad}$ ) in the cytoplasm and translocation of $\mathrm{Mcl}-1$ to the mitochondria, and/or inhibit Mcl-1 degradation. GMCSF predominantly influences proteasomal degradation of $\mathrm{Mcl}-1$. Blockade of survival signalling circuits by MAPK inhibitors, lipoxin $A_{4}$, or aspirin-triggered 15 -epi-LXA $A_{4}$ redirects neutrophils to apoptosis by decreasing $\mathrm{Mcl}-1$ level. Likewise, the CDK inhibitor roscovitine evokes drops in $\mathrm{Mcl}-1$ through yet undefined mechanisms. Therapeutic induction of neutrophil apoptosis through modulation of $\mathrm{Mcl}-1$ expression would contribute to clearance of emigrated neutrophils, thereby enhancing the resolution of inflammation. 
whereas induction of apoptosis in neutrophils exerts anti-inflammatory actions and enhances the resolution of inflammation. We have highlighted the importance of Mcl-1 in neutrophil survival and attempted to review the multiple levels of control of Mcl-1 expression and function as well as the intricate interplay between survival and proapoptosis signaling circuits that determine the fate of neutrophils (Figure 1). We also discussed that old and emerging therapeutic strategies to target neutrophils appear to act via modulation of Mcl-1. Small molecules affecting the expression or function of Mcl-1 in a neutrophil-specific fashion appear to be promising tools to dampen the harmful excesses of inflammation.

\section{ACKNOWLEDGMENTS}

This work was supported by Grants from the Lymphoma and Leukemia Society of Canada (E. Milot) and MOP-67054 and MOP-97742 from the Canadian Institutes of Health Research (J. G. Filep).

\section{REFERENCES}

[1] J. Savill, I. Dransfield, C. Gregory, and C. Haslett, "A blast from the past: clearance of apoptotic cells regulates immune responses," Nature Reviews Immunology, vol. 2, no. 12, pp. 965-975, 2002.

[2] D. W. Gilroy, T. Lawrence, M. Perretti, and A. G. Rossi, "Inflammatory resolution: new opportunities for drug discovery," Nature Reviews Drug Discovery, vol. 3, no. 5, pp. 401-416, 2004.

[3] H. R. Luo and F. Loison, "Constitutive neutrophil apoptosis: mechanisms and regulation," The American Journal of Hematology, vol. 83, no. 4, pp. 288-295, 2008.

[4] H. U. Simon, "Neutrophil apoptosis pathways and their modifications in inflammation," Immunological Reviews, vol. 193, pp. 101-110, 2003.

[5] D. El Kebir and J. G. Filep, "Role of neutrophil apoptosis in the resolution of inflammation," TheScientificWorldJOURNAL, vol. 10, pp. 1731-1748, 2010.

[6] C. Nathan, "Neutrophils and immunity: challenges and opportunities," Nature Reviews Immunology, vol. 6, no. 3, pp. 173-182, 2006.

[7] W. M. Nauseef, "How human neutrophils kill and degrade microbes: an integrated view," Immunological Reviews, vol. 219, no. 1, pp. 88-102, 2007.

[8] C. Elbim, P.D . Katsikis, and J. Estaquier, "Neutrophil apoptosis during viral infections," The Open Virology Journal, vol. 3, pp. 52-59, 2009.

[9] M. J. Ramirez, E. Titos, J. Claria, M. Navasa, and J. Rodes J. Fernandez, "Increased apoptosis dependent on caspase-3 activity in polymorphonuclear leukocytes from patients with cirrhosis and ascites," Journal of Hepatology, vol. 41, pp. 44-48, 2004.

[10] J. S. Savill, A. H. Wyllie, J. E. Henson, M. J. Walport, P. M. Henson, and C. Haslett, "Macrophage phagocytosis of aging neutrophils in inflammation. Programmed cell death in the neutrophil leads to its recognition by macrophages," Journal of Clinical Investigation, vol. 83, no. 3, pp. 865-875, 1989.

[11] D. El Kebir, L. Jozsef, W. Pan, and J. G. Filep, "Myeloperoxidase delays neutrophil apoptosis through CD11b/CD18 integrins and prolongs inflammation," Circulation Research, vol. 103, no. 4, pp. 352-359, 2008.

[12] H. Jonsson, P. Allen, and S. L. Peng, "Inflammatory arthritis requires Foxo3a to prevent Fas ligand-induced neutrophil apoptosis," Nature Medicine, vol. 11, no. 6, pp. 666-671, 2005.

[13] S. Khanna, S. Biswas, Y. Shang et al., "Macrophage dysfunction impairs resolution of inflammation in the wounds of diabetic mice," PLoS One, vol. 5, no. 3, Article ID e9539, 2010.

[14] C. N. Serhan, S. D. Brain, C. D. Buckley et al., "Resolution of inflammation: state of the art, definitions and terms," FASEB Journal, vol. 21, no. 2, pp. 325-332, 2007.

[15] C. N. Serhan, N. Chiang, and T. E. Van Dyke, "Resolving inflammation: dual anti-inflammatory and proresolution lipid mediators," Nature Reviews Immunology, vol. 8, no. 5, pp. 349-361, 2008.

[16] S. W. Edwards, M. Derouet, M. Howse, and R. J. Moots, "Regulation of neutrophil apoptosis by Mcl-1," Biochemical Society Transactions, vol. 32, no. 3, pp. 489-492, 2004.

[17] I. Dzhagalov, A. S. John, and Y. W. He, "The antiapoptotic protein Mcl-1 is essential for the survival of neutrophils but not macrophages," Blood, vol. 109, no. 4, pp. 1620-1626, 2007. 
[18] D. A. Moulding, C. Akgul, M. Derouet, M. R. H. White, and S. W. Edwards, "BCL-2 family expression in human neutrophils during delayed and accelerated apoptosis," Journal of Leukocyte Biology, vol. 70, no. 5, pp. 783-792, 2001.

[19] R. W. Craig, "MCL1 provides a window on the role of the BCL2 family in cell proliferation, differentiation and tumorigenesis," Leukemia, vol. 16, no. 4, pp. 444-454, 2002.

[20] J. T. Dancey, K. A. Deubelbeiss, C. A. Harker, and C.A. Finch, "Neutrophil kinetics in man," Journal of Clinical Investigation, vol. 58, no. 3, pp. 705-715, 1976.

[21] S. Basu, G. Hodgson, M. Katz, and A. R. Dunn, "Evaluation of role of G-CSF in the production, survival, and release of neutrophils from bone marrow into circulation," Blood, vol. 100, no. 3, pp. 854-861, 2002.

[22] J. Pillay, I. den Braber, N. Vrisekoop et al., "In vivo labeling with ${ }^{2} \mathrm{H}_{2} \mathrm{O}$ reveals a human neutrophil lifespan of 5.4 days," Blood, vol. 116, no. 4, pp. 625-627, 2010.

[23] C. Martin, P. C. E. Burdon, G. Bridger, J. C. Gutierrez-Ramos, T. J. Williams, and S. M. Rankin, "Chemokines acting via CXCR2 and CXCR4 control the release of neutrophils from the bone marrow and their return following senescence," Immunity, vol. 19, no. 4, pp. 583-593, 2003.

[24] R. W. G. Watson, O. D. Rotstein, A. B. Nathens, J. Parodo, and J. C. Marshall, "Neutrophil apoptosis modulated by endothelial transmigration and adhesion molecules engagement," Journal of Immunology, vol. 158, no. 2, pp. 945-953, 1997.

[25] A. Lee, M. K. Whyte, and C. Haslett, "Inhibition of apoptosis and prolongation of neutrophil functional longevity by inflammatory mediators," Journal of Leukocyte Biology, vol. 54, no. 4, pp. 283-288, 1993.

[26] F. Colotta, F. Re, N. Polentarutti, S. Sozzani, and A. Mantovani, "Modulation of granulocyte survival and programmed cell death by cytokines and bacterial products," Blood, vol. 80, no. 8, pp. 2012-2020, 1992.

[27] D. El Kebir, L. József, T. Khreiss et al., "Aspirin-triggered lipoxins override the apoptosis-delaying action of serum amyloid A in human neutrophils: a novel mechanism for resolution of inflammation," Journal of Immunology, vol. 179, no. 1, pp. 616-622, 2007.

[28] T. Khreiss, L. József, S. Hossain, J. S. D. Chan, L. A. Potempa, and J. G. Filep, "Loss of pentameric symmetry of C-reactive protein is associated with delayed apoptosis of human neutrophils," Journal of Biological Chemistry, vol. 277, no. 43, pp. 40775-40781, 2002.

[29] S. A. Renshaw, J. S. Parmar, V. Singleton et al., "Acceleration of human neutrophil apoptosis by TRAIL," Journal of Immunology, vol. 170, no. 2, pp. 1027-1033, 2003.

[30] T. N. Mayadas and X. Cullere, "Neutrophil $\beta_{2}$ integrins: moderators of life or death decisions," Trends in Immunology, vol. 26, no. 7, pp. 388-395, 2005.

[31] J. Savill, I. Dransfield, N. Hogg, and C. Haslett, "Vitronectin receptor-mediated phagocytosis of cells undergoing apoptosis," Nature, vol. 343, no. 6254, pp. 170-173, 1990.

[32] L. Allen, D. H. Dockrell, T. Pattery et al., "Pyocyanin production by Pseudomonas aeruginosa induces neutrophil apoptosis and impairs neutrophil-mediated host defenses in vivo," Journal of Immunology, vol. 174, no. 6, pp. 3643-3649, 2005.

[33] M. L. Colamussi, M. R. White, E. Crouch, and K. L. Hartshorn, "Influenza A virus accelerates neutrophil apoptosis and markedly potentiates apoptotic effects of bacteria," Blood, vol. 93, no. 7, pp. 2395-2403, 1999.

[34] P. A. Courtney, A. D. Crockard, K. Williamson, A. E. Irvine, R. J. Kennedy, and A. L. Bell, "Increased apoptotic peripheral blood neutrophils in systemic lupus erythematosus: relations with disease activity, antibodies to double stranded DNA, and neutropenia," Annals of the Rheumatic Diseases, vol. 58, no. 5, pp. 309-314, 1999.

[35] W. Ertel, M. Keel, M. Infanger, U. Ungethüm, U. Steckholzer, and O. Trentz, "Circulating mediators in serum of injured patients with septic complications inhibit neutrophil apoptosis through up-regulation of protein- tyrosine phosphorylation," Journal of Trauma, vol. 44, no. 5, pp. 767-776, 1998.

[36] D. Chitnis, C. Dickerson, A. M. Munster, and R. A. Winchurch, "Inhibition of apoptosis in polymorphonuclear neutrophils from burn patients," Journal of Leukocyte Biology, vol. 59, no. 6, pp. 835-839, 1996.

[37] G. Matute-bello, W. C. Liles, F. Radella II et al., "Neutrophil apoptosis in the acute respiratory distress syndrome," The American Journal of Respiratory and Critical Care Medicine, vol. 156, no. 6, pp. 1969-1977, 1997.

[38] C. D. Garlichs, S. Eskafi, I. Cicha et al., "Delay of neutrophil apoptosis in acute coronary syndromes,” Journal of Leukocyte Biology, vol. 75, no. 5, pp. 828-835, 2004. 
[39] V. Brown, J. S. Elborn, J. Bradley, and M. Ennis, "Dysregulated apoptosis and NFkappaB expression in COPD subjects," Respiratory research, vol. 10, p. 24, 2009.

[40] C. A. Lindemans, P. J. Coffer, I. M. M. Schellens, P. M. A. De Graaff, J. L. L. Kimpen, and L. Koenderman, "Respiratory syncytial virus inhibits granulocyte apoptosis through a phosphatidylinositol 3-kinase and NF- $\kappa$ Bdependent mechanism," Journal of Immunology, vol. 176, no. 9, pp. 5529-5537, 2006.

[41] S. H. Wong, N. Francis, H. Chahal et al., "Lactoferrin is a survival factor for neutrophils in rheumatoid synovial fluid," Rheumatology, vol. 48, no. 1, pp. 39-44, 2009.

[42] K. Christenson, L. Björkman, C. Töngemo, and J. Bylund, "Serum amyloid A inhibits apoptosis of human neutrophils via a P2X7-sensitive pathway independent of formyl peptide receptor-like 1," Journal of Leukocyte Biology, vol. 83, no. 1, pp. 139-148, 2008.

[43] S. O’Neill, A. J. O’Neill, E. Conroy, H. R. Brady, J. M. Fitzpatrick, and R. W. G. Watson, "Altered caspase expression results in delayed neutrophil apoptosis in acute pancreatitis," Journal of Leukocyte Biology, vol. 68, no. 1, pp. 15-20, 2000.

[44] E. I. Finkelstein, M. Nardini, and A. Van Der Vliet, "Inhibition of neutrophil apoptosis by acrolein: a mechanism of tobacco-related lung disease?" The American Journal of Physiology, vol. 281, no. 3, pp. L732-L739, 2001.

[45] T. Laskay, G. van Zandbergen, and W. Solbach, "Neutrophil granulocytes as host cells and transport vehicles for intracellular pathogens: apoptosis as infection-promoting factor," Immunobiology, vol. 213, no. 3-4, pp. 183-191, 2008.

[46] J. Rupp, L. Pfleiderer, C. Jugert et al., "Chlamydia pneumoniae hides inside apoptotic neutrophils to silently infect and propagate in macrophages," PLoS One, vol. 4, no. 6, Article ID e6020, 2009.

[47] D. A. Moulding, C. Akgul, M. Derouet, M. R. H. White, and S. W. Edwards, "BCL-2 family expression in human neutrophils during delayed and accelerated apoptosis," Journal of Leukocyte Biology, vol. 70, no. 5, pp. 783-792, 2001.

[48] C. Akgul, D. A. Moulding, and S. W. Edwards, "Molecular control of neutrophil apoptosis," FEBS Letters, vol. 487, no. 3, pp. 318-322, 2001.

[49] A. Hamasaki, F. Sendo, K. Nakayama et al., "Accelerated neutrophil apoptosis in mice lacking A1-a, a subtype of the bcl-2-related A1 gene," Journal of Experimental Medicine, vol. 188, no. 11, pp. 1985-1992, 1998.

[50] D. A. Moulding, J. A. Quayle, C. Anthony Hart, and S. W. Edwards, "Mcl-1 expression in human neutrophils: regulation by cytokines and correlation with cell survival," Blood, vol. 92, no. 7, pp. 2495-2502, 1998.

[51] T. Kato, H. Kutsuna, N. Oshitani, and S. Kitagawa, "Cyclic AMP delays neutrophil apoptosis via stabilization of Mcl-1," FEBS Letters, vol. 580, no. 19, pp. 4582-4586, 2006.

[52] S. J. Leuenroth, P. S. Grutkoski, A. Ayala, and H. H. Simms, "Suppression of PMN apoptosis by hypoxia is dependent on Mcl-1 and MAPK activity," Surgery, vol. 128, no. 2, pp. 171-177, 2000.

[53] D. A. Moulding, R. V. Giles, D. G. Spiller, M. R. H. White, D. M. Tidd, and S. W. Edwards, "Apoptosis is rapidly triggered by antisense depletion of MCL-1 in differentiating U937 cells," Blood, vol. 96, no. 5, pp. 1756-1763, 2000.

[54] S. Catarzi, T. Marcucci, L. Papucci et al., "Apoptosis and bax, Bcl-2, Mcl-1 expression in neutrophils of Crohn's disease patients," Inflammatory Bowel Diseases, vol. 14, no. 6, pp. 819-825, 2008.

[55] N. Fotouhi-Ardakani, D. El Kebir, N. Pierre-Charles et al., "Role for myeloid nuclear differentiation antigen in the regulation of neutrophil apoptosis during sepsis," The American Journal of Respiratory and Critical Care Medicine, vol. 182, no. 3, pp. 341-350, 2010.

[56] S. Fox, A. E. Leitch, R. Duffin, C. Haslett, and A. G. Rossi, "Neutrophil apoptosis: relevance to the innate immune response and inflammatory disease," Journal of Innate Immunity, vol. 2, no. 3, pp. 216-227, 2010.

[57] J. G. Filep and D. El Kebir, "Neutrophil apoptosis: a target for enhancing the resolution of inflammation," Journal of Cellular Biochemistry, vol. 108, no. 5, pp. 1039-1046, 2009.

[58] B. Zhang, J. Hirahashi, X. Cullere, and T. N. Mayadas, "Elucidation of molecular events leading to neutrophil apoptosis following phagocytosis: cross-talk between caspase 8 , reactive oxygen species, and MAPK/ERK activation," Journal of Biological Chemistry, vol. 278, no. 31, pp. 28443-28454, 2003.

[59] A. Kanayama and Y. Miyamoto, "Apoptosis triggered by phagocytosis-related oxidative stress through FLIPS down-regulation and JNK activation,” Journal of Leukocyte Biology, vol. 82, no. 5, pp. 1344-1352, 2007. 
[60] R. D. Ye, F. Boulay, M. W. Ji et al., "International union of basic and clinical pharmacology. LXXIII. Nomenclature for the formyl peptide receptor (FPR) family," Pharmacological Reviews, vol. 61, no. 2, pp. 119-161, 2009.

[61] N. Chiang, C. N. Serhan, S. -E. Dahlén et al., "The lipoxin receptor ALX: potent ligand-specific and stereoselective actions in vivo," Pharmacological Reviews, vol. 58, no. 3, pp. 463-487, 2006.

[62] M. Perretti, N. Chiang, M. La et al., "Endogenous lipid- and peptide-derived anti-inflammatory pathways generated with glucocorticoid and aspirin treatment activate the lipoxin $\mathrm{A}_{4}$ receptor," Nature Medicine, vol. 8, no. 11, pp. 1296-1302, 2002.

[63] I. Nagaoka, H. Tamura, and M. Hirata, "An antimicrobial cathelicidin peptide, human CAP18/LL-37, suppresses neutrophil apoptosis via the activation of formyl-peptide receptor-like 1 and P2X 7 ," Journal of Immunology, vol. 176, no. 5, pp. 3044-3052, 2006.

[64] F. Altznauer, S. Conus, A. Cavalli, G. Folkers, and H. U. Simon, "Calpain-1 regulates Bax and subsequent Smac-dependant caspase-3 activation in neutrophil apoptosis," Journal of Biological Chemistry, vol. 279, no. 7, pp. 5947-5957, 2004.

[65] J. Michels, P. W. M. Johnson, and G. Packham, "Molecules in focus. Mcl-1," The International Journal of Biochemistry \& Cell Biology, vol. 37, pp. 267-271, 2005.

[66] L. W. Thomas, C. Lam, and S. W. Edwards, "Mcl-1; the molecular regulation of protein function," FEBS Letters, vol. 584, no. 14, pp. 2981-2989, 2010.

[67] A. Gross, J. M. McDonnell, and S. J. Korsmeyer, "BCL-2 family members and the mitochondria in apoptosis," Genes and Development, vol. 13, no. 15, pp. 1899-1911, 1999.

[68] T. Yang, K. M. Kozopas, and R. W. Craig, "The intracellular distribution and pattern of expression of Mcl-1 overlap with, but are not identical to, those of Bcl-2," Journal of Cell Biology, vol. 128, no. 6, pp. 1173-1184, 1995.

[69] R. W. Craig, "MCL1 provides a window on the role of the BCL2 family in cell proliferation, differentiation and tumorigenesis," Leukemia, vol. 16, no. 4, pp. 444-454, 2002.

[70] A. Cuconati, C. Mukherjee, D. Perez, and E. White, "DNA damage response and MCL-1 destruction initiate apoptosis in adenovirus-infected cells," Genes and Development, vol. 17, no. 23, pp. 2922-2932, 2003.

[71] J. C. Reed, "Proapoptotic multidomain Bcl-2/Bax-family proteins: mechanisms, physiological roles, and therapeutic opportunities," Cell Death and Differentiation, vol. 13, no. 8, pp. 1378-1386, 2006.

[72] D. Brenner and T. W. Mak, "Mitochondrial cell death effectors," Current Opinion in Cell Biology, vol. 21, no. 6, pp. 871-877, 2009.

[73] N. A. Maianski, J. Geissler, S. M. Srinivasula, E. S. Alnemri, D. Roos, and T. W. Kuijpers, "Functional characterization of mitochondria in neutrophils: a role restricted to apoptosis," Cell Death and Differentiation, vol. 11, no. 2, pp. 143-153, 2004.

[74] C. Akgul, P. C. Turner, M. R. H. White, and S. W. Edwards, "Functional analysis of the human MCL-1 gene," Cellular and Molecular Life Sciences, vol. 57, no. 4, pp. 684-691, 2000.

[75] M. Derouet, L. Thomas, A. Cross, R. J. Moots, and S. W. Edwards, "Granulocyte macrophage colonystimulating factor signaling and proteasome inhibition delay neutrophil apoptosis by increasing the stability of Mcl-1," Journal of Biological Chemistry, vol. 279, no. 26, pp. 26915-26921, 2004.

[76] S. Negrotto, E. Malaver, M. E. Alvarez et al., "Aspirin and salicylate suppress polymorphonuclear apoptosis delay mediated by proinflammatory stimuli," Journal of Pharmacology and Experimental Therapeutics, vol. 319, no. 2, pp. 972-979, 2006.

[77] A. Drewniak, B. J. van Raam, J. Geissler et al., "Changes in gene expression of granulocytes during in vivo granulocyte colony-stimulating factor/dexamethasone mobilization for transfusion purposes," Blood, vol. 113, no. 23, pp. 5979-5998, 2009.

[78] Y. Kasahara, K. Iwai, A. Yachie et al., "Involvement of reactive oxygen intermediates in spontaneous and CD95(Fas/APO-1)-mediated apoptosis of neutrophils," Blood, vol. 89, no. 5, pp. 1748-1753, 1997.

[79] S. Hannah, K. Mecklenburgh, I. Rahman et al., "Hypoxia prolongs neutrophil survival in vitro," FEBS Letters, vol. 372, no. 2-3, pp. 233-237, 1995.

[80] C. Ward, A. Walker, I. Dransfield, C. Haslett, and A. G. Rossi, "Regulation of granulocyte apoptosis by NF- $\kappa$ B," Biochemical Society Transactions, vol. 32, no. 3, pp. 465-467, 2004. 
[81] R. Croxton, Y. Ma, L. Song, E. B. Haura, and W. D. Cress, "Direct repression of the Mcl-1 promoter by E2F1," Oncogene, vol. 21, no. 9, pp. 1359-1369, 2002.

[82] J. H. Kim, S. H. Sim, H. J. Ha, J. J. Ko, K. Lee, and J. Bae, "MCL-1ES, a novel variant of MCL-1, associates with MCL-1L and induces mitochondrial cell death," FEBS Letters, vol. 583, no. 17, pp. 2758-2764, 2009.

[83] J. Bae, C. P. Leo, Sheau Yu Hsu, and A. J. W. Hsueh, "MCL-1S, a splicing variant of the antiapoptotic BCL-2 family member MCL-1, encodes a proapoptotic protein possessing only the BH3 domain," Journal of Biological Chemistry, vol. 275, no. 33, pp. 25255-25261, 2000.

[84] H. M. Marriott, C. D. Bingle, R. C. Read et al., "Dynamic changes in Mcl-1 expression regulate macrophage viability or commitment to apoptosis during bacterial clearance," Journal of Clinical Investigation, vol. 115, no. 2, pp. 359-368, 2005.

[85] J. L. Mott, S. Kobayashi, S. F. Bronk, and G. J. Gores, "mir-29 regulates Mcl-1 protein expression and apoptosis," Oncogene, vol. 26, no. 42, pp. 6133-6140, 2007.

[86] S. Kobayashi, S. H. Lee, X. W. Meng et al., "Serine 64 phosphorylation enhances the antiapoptotic function of Mcl-1," Journal of Biological Chemistry, vol. 282, no. 25, pp. 18407-18417, 2007.

[87] Q. Ding, L. Huo, J. Y. Yang et al., "Down-regulation of myeloid cell leukemia-1 through inhibiting Erk/Pin 1 pathway by sorafenib facilitates chemosensitization in breast cancer," Cancer Research, vol. 68, no. 15, pp. 6109-6117, 2008.

[88] Q. Ding, X. He, J. M. Hsu et al., "Degradation of Mcl-1 by $\beta$-TrCP mediates glycogen synthase kinase 3-induced tumor suppression and chemosensitization," Molecular and Cellular Biology, vol. 27, no. 11, pp. 4006-4017, 2007.

[89] U. Maurer, C. Charvet, A. S. Wagman, E. Dejardin, and D. R. Green, "Glycogen synthase kinase-3 regulates mitochondrial outer membrane permeabilization and apoptosis by destabilization of MCL-1," Molecular Cell, vol. 21, no. 6, pp. 749-760, 2006.

[90] C. Morel, S. M. Carlson, F. M. White, and R. J. Davis, "Mcl-1 integrates the opposing actions of signaling pathways that mediate survival and apoptosis," Molecular and Cellular Biology, vol. 29, no. 14, pp. 3845-3852, 2009.

[91] M. Alvarado-Kristensson, F. Melander, K. Leandersson, L. Rönnstrand, C. Wernstedt, and T. Andersson, "p38MAPK signals survival by phosphorylation of caspase-8 and caspase-3 in human neutrophils," Journal of Experimental Medicine, vol. 199, no. 4, pp. 449-458, 2004.

[92] J. B. Klein, M. J. Rane, J. A. Scherzer et al., "Granulocyte-macrophage colony-stimulating factor delays neutrophil constitutive apoptosis through phosphoinositide 3-kinase and extracellular signal-regulated kinase pathways," Journal of Immunology, vol. 164, no. 8, pp. 4286-4291, 2000.

[93] K. Fujise, D. Zhang, J. L. Liu, and E. T. H. Yeh, "Regulation of apoptosis and cell cycle progression by MCL1. Differential role of proliferating cell nuclear antigen," Journal of Biological Chemistry, vol. 275, no. 50, pp. 39458-39465, 2000.

[94] S. Jamil, R. Sobouti, P. Hojabrpour, M. Raj, J. Kast, and V. Duronio, "A proteolytic fragment of Mcl-1 exhibits nuclear localization and regulates cell growth by interaction with Cdk1," Biochemical Journal, vol. 387, no. 3, pp. 659-667, 2005.

[95] M. Germain and V. Duronio, "The N terminus of the anti-apoptotic BCL-2 homologue MCL-1 regulates its localization and function," Journal of Biological Chemistry, vol. 282, no. 44, pp. 32233-32242, 2007.

[96] S. J. Gardai, D. A. Hildeman, S. K. Frankel et al., "Phosphorylation of Bax ser184 by Akt regulates its activity and apoptosis in neutrophils," Journal of Biological Chemistry, vol. 279, no. 20, pp. 21085-21095, 2004.

[97] J. Y. J. Wang, "Nucleo-cytoplasmic communication in apoptotic response to genotoxic and inflammatory stress," Cell Research, vol. 15, no. 1, pp. 43-48, 2005.

[98] M. Mihara, S. Erster, A. Zaika et al., "p53 has a direct apoptogenic role at the mitochondria," Molecular Cell, vol. 11, no. 3, pp. 577-590, 2003.

[99] P. Dumont, J. I. J. Leu, A. C. Della Pietra, D. L. George, and M. Murphy, "The codon 72 polymorphic variants of p53 have markedly different apoptotic potential," Nature Genetics, vol. 33, no. 3, pp. 357-365, 2003.

[100] J. E. Chipuk, U. Maurer, D. R. Green, and M. Schuler, "Pharmacologic activation of p53 elicits Bax-dependent apoptosis in the absence of transcription," Cancer Cell, vol. 4, no. 5, pp. 371-381, 2003.

[101] J. E. Chipuk and D. R. Green, "Cytoplasmic p53: bax and forward," Cell Cycle, vol. 3, no. 4, pp. 429-431, 2004. 
[102] Y. Zhang and L. Wang, "Nuclear receptor small heterodimer partner in apoptosis signaling and liver cancer," Cancers, vol. 3, no. 1, pp. 198-212, 2011.

[103] V. Witko-Sarsat, J. Mocek, D. Bouayad et al., "Proliferating cell nuclear antigen acts as a cytoplasmic platform controlling human neutrophil survival," Journal of Experimental Medicine, vol. 207, no. 12, pp. 2631-2645, 2010.

[104] S. N. Naryzhny, "Proliferating cell nuclear antigen: a proteomics view," Cellular and Molecular Life Sciences, vol. 65, no. 23, pp. 3789-3808, 2008.

[105] D. Choubey and R. Panchanathan, "Interferon-inducible Ifi200-family genes in systemic lupus erythematosus," Immunology Letters, vol. 119, no. 1-2, pp. 32-41, 2008.

[106] Q. Zhong, W. Gao, F. Du, and X. Wang, "Mule/ARF-BP1, a BH3-only E3 ubiquitin ligase, catalyzes the polyubiquitination of Mcl-1 and regulates apoptosis," Cell, vol. 121, no. 7, pp. 1085-1095, 2005.

[107] M. Schwickart, X. Huang, J. R. Lill et al., "Deubiquitinase USP9X stabilizes MCL1 and promotes tumour cell survival," Nature, vol. 463, no. 7277, pp. 103-107, 2010.

[108] J. G. Clohessy, J. Zhuang, and H. J. M. Brady, "Characterisation of Mcl-1 cleavage during apoptosis of haematopoietic cells," The British Journal of Haematology, vol. 125, no. 5, pp. 655-665, 2004.

[109] C. Weng, Y. Li, D. Xu, Y. Shi, and H. Tang, "Specific cleavage of Mcl-1 by caspase-3 in tumor necrosis factorrelated apoptosis-inducing ligand (TRAIL)-induced apoptosis in Jurkat leukemia T cells," Journal of Biological Chemistry, vol. 280, no. 11, pp. 10491-10500, 2005.

[110] D. J. Wardle, J. Burgon, I. Sabroe, C. D. Bingle, M. K.B. Whyte, and S. A. Renshaw, "Effective caspase inhibition blocks neutrophil apoptosis and reveals Mcl-1 as both a regulator and a target of neutrophil caspase activation," PLoS One, vol. 6, no. 1, Article ID e15768, 2011.

[111] K. Miles, D. J. Clarke, W. Lu et al., "Dying and necrotic neutrophils are anti-inflammatory secondary to the release of alpha-defensins," Journal of Immunology, vol. 183, no. 3, pp. 2122-2132, 2009.

[112] A. Ariel, G. Fredman, Y. P. Sun et al., "Apoptotic neutrophils and T cells sequester chemokines during immune response resolution through modulation of CCR5 expression," Nature Immunology, vol. 7, no. 11, pp. 12091216, 2006.

[113] Y. Ren, Y. Xie, G. Jiang et al., "Apoptotic cells protect mice against lipopolysaccharide-induced shock," Journal of Immunology, vol. 180, no. 7, pp. 4978-4985, 2008.

[114] P. J. Murray and T. A. Wynn, "Obstacles and opportunities for understanding macrophage polarization," Journal of Leukocyte Biology, vol. 89, no. 4, pp. 557-563, 2011.

[115] V. Pinho, R. D. C. Russo, F. A. Amaral et al., "Tissue- and stimulus-dependent role of phosphatidylinositol 3-kinase isoforms for neutrophil recruitment induced by chemoattractants in vivo," Journal of Immunology, vol. 179, no. 11, pp. 7891-7898, 2007.

[116] D. A. Sawatzky, D. A. Willoughby, P. R. Colville-Nash, and A. G. Rossi, "The involvement of the apoptosismodulating proteins ERK 1/2, Bcl-x L and Bax in the resolution of acute inflammation in vivo," The American Journal of Pathology, vol. 168, no. 1, pp. 33-41, 2006.

[117] M. Derouet, L. Thomas, D. A. Moulding et al., "Sodium salicylate promotes neutrophil apoptosis by stimulating caspase-dependent turnover of Mcl-1," Journal of Immunology, vol. 176, no. 2, pp. 957-965, 2006.

[118] L. P. Sousa, F. Lopes, D. M. Silva et al., "PDE4 inhibition drives resolution of neutrophilic inflammation by inducing apoptosis in a PKA-PI3K/Akt-dependent and NF- $\kappa$ B-independent manner," Journal of Leukocyte Biology, vol. 87, no. 5, pp. 895-904, 2010.

[119] D. El Kebir, L. József, W. Pan et al., "15-epi-lipoxin A4 inhibits myeloperoxidase signaling and enhances resolution of acute lung injury," The American Journal of Respiratory and Critical Care Medicine, vol. 180, no. 4, pp. 311-319, 2009.

[120] V. Brovkovych, X. P. Gao, E. Ong et al., "Augmented inducible nitric oxide synthase expression and increased NO production reduce sepsis-induced lung injury and mortality in myeloperoxidase-null mice," The American Journal of Physiology, vol. 295, no. 1, pp. L96-L103, 2008.

[121] R. A. Matthijsen, D. Huugen, N. T. Hoebers et al., "Myeloperoxidase is critically involved in the induction of organ damage after renal ischemia reperfusion," The American Journal of Pathology, vol. 171, no. 6, pp. 1743-1752, 2007.

[122] K. Fukunaga, P. Kohli, C. Bonnans, L. E. Fredenburgh, and B. D. Levy, "Cyclooxygenase 2 plays a pivotal role in the resolution of acute lung injury," Journal of Immunology, vol. 174, no. 8, pp. 5033-5039, 2005. 
[123] A. Planagumà, M. A. Pfeffer, G. Rubin et al., "Lovastatin decreases acute mucosal inflammation via 15-epilipoxin A4," Mucosal Immunology, vol. 3, no. 3, pp. 270-279, 2010.

[124] L. József, C. Zouki, N. A. Petasis, C. N. Serhan, and J. G. Filep, "Lipoxin A4 and aspirin-triggered 15-epilipoxin $\mathrm{A}_{4}$ inhibit peroxynitrite formation, NF- $\kappa \mathrm{B}$ and AP-1 activation, and IL-8 gene expression in human leukocytes," Proceedings of the National Academy of Sciences of the United States of America, vol. 99, no. 20, pp. 13266-13271, 2002.

[125] D. Iglesias-Serret, M. Pique, M. Barragan et al., "Aspirin induces apoptosis in human leukemia cells independently of NF- $\kappa$ B and MAPKs through alteration of the Mcl-1/Noxa balance," Apoptosis, vol. 15, pp. 219-229, 2010.

[126] C. N. Serhan, S. Hong, K. Gronert et al., "Resolvins: a family of bioactive products of omega-3 fatty acid transformation circuits initiated by aspirin treatment that counter proinflammation signals," Journal of Experimental Medicine, vol. 196, no. 8, pp. 1025-1037, 2002.

[127] H. Seki, K. Fukunaga, M. Arita et al., "The anti-inflammatory and proresolving mediator resolving E1 protects mice from bacterial pneumonia and acute lung injury," The Journal of Immunology, vol. 184, pp. 836-843, 2010.

[128] M. Spite, L. V. Norling, L. Summers et al., "Resolvin D2 is a potent regulator of leukocytes and controls microbial sepsis," Nature, vol. 461, no. 7268, pp. 1287-1291, 2009.

[129] A. G. Rossi, D. A. Sawatzky, A. Walker et al., "Cyclin-dependent kinase inhibitors enhance the resolution of inflammation by promoting inflammatory cell apoptosis," Nature Medicine, vol. 12, no. 9, pp. 1056-1064, 2006.

[130] J. L. Rosales, J. D. Ernst, J. Hallows, and K. Y. Lee, "GTP-dependent secretion from neutrophils is regulated by Cdk5," Journal of Biological Chemistry, vol. 279, no. 52, pp. 53932-53936, 2004.

[131] U. Koedel, T. Frankenberg, S. Kirschnek et al., "Apoptosis is essential for neutrophil functional shutdown and determines tissue damage in experimental pneumococcal meningitis," PLoS Pathogens, vol. 5, no. 5, Article ID e1000461, 2009.

[132] S. Moriceau, G. Lenoir, and V. Witko-Sarsat, "In cystic fibrosis homozygotes and heterozygotes, neutrophil apoptosis is delayed and modulated by diamide or roscovitine: evidence for an innate neutrophil disturbance," Journal of Innate Immunity, vol. 2, no. 3, pp. 260-266, 2010.

[133] A. Paunel-Görgülü, M. Zörnig, T. Lögters et al., "Mcl-1-mediated impairment of the intrinsic apoptosis pathway in circulating neutrophils from critically ill patients can be overcome by Fas stimulation," Journal of Immunology, vol. 183, no. 10, pp. 6198-6206, 2009.

[134] J. T. Opferman, A. Letai, C. Beard, M. D. Sorcinelli, C. C. Ong, and S. J. Korsmeyer, "Development and maintenance of B and T lymphocytes requires antiapoptotic MCL-1," Nature, vol. 426, no. 6967, pp. 671-676, 2003.

[135] J. T. Opferman, H. Iwasaki, C. C. Ong et al., "Obligate role of anti-apoptotic MCL-1 in the survival of hematopoietic stem cells," Science, vol. 307, no. 5712, pp. 1101-1104, 2005.

\section{This article should be cited as follows:}

Eric Milot and János G. Filep , "Regulation of Neutrophil Survival/Apoptosis by Mcl-1," TheScientificWorldJOURNAL, vol. 11, pp. 1948-1962, 2011. 


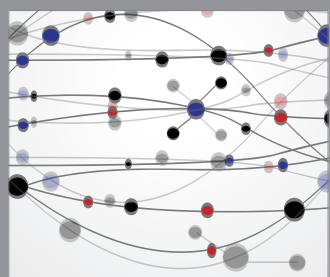

The Scientific World Journal
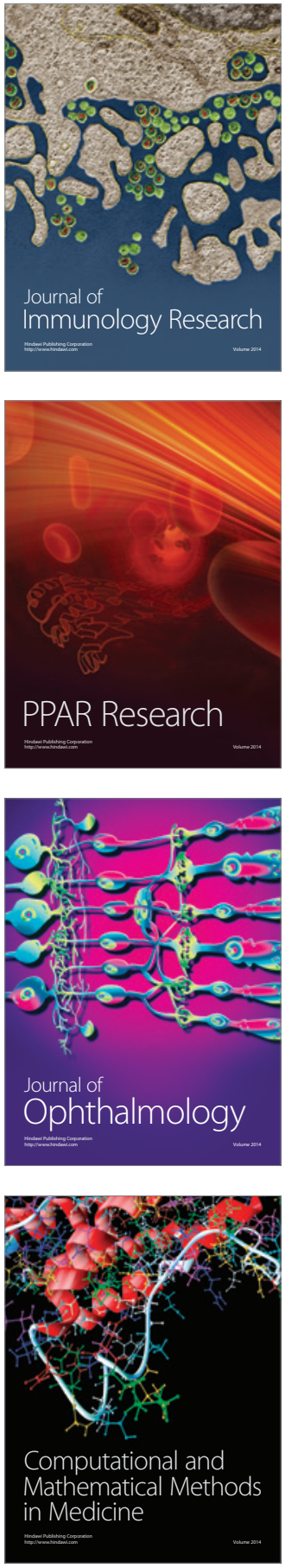

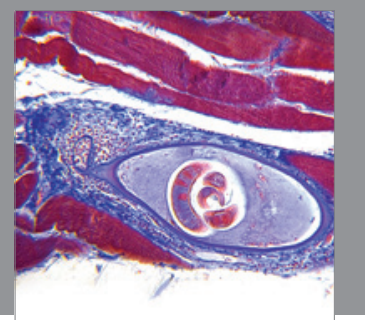

Gastroenterology

Research and Practice
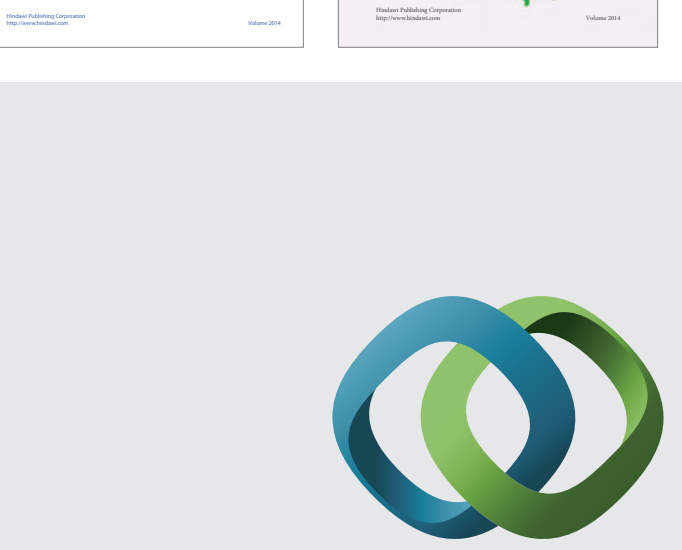

\section{Hindawi}

Submit your manuscripts at

http://www.hindawi.com
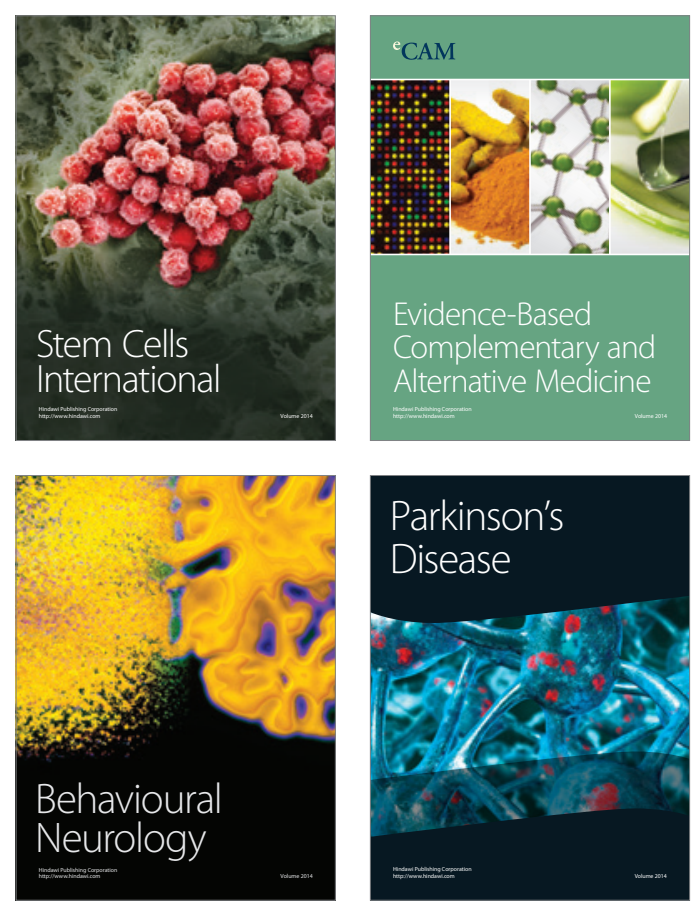

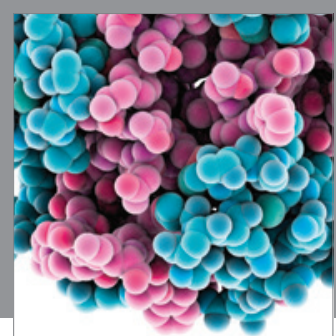

Journal of
Diabetes Research

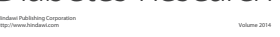

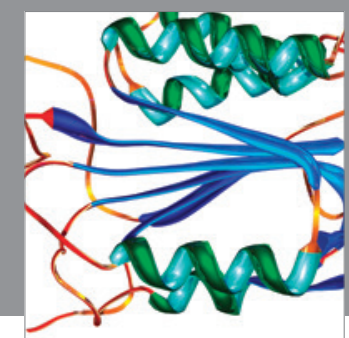

Disease Markers
